of unemployment at 6 or 24 months. All models were adjusted for age, gender, level of education and country of origin. Gender interactions were examined.

Results 3969 participants were employed or unemployed and seeking work at baseline and at 6 months. $6 \%(n=221)$ had major depression at 12 months. Participants who were employed baseline but unemployed at 6 months compared to those employed at both time points had adjusted RRR of 1.63 (95\% CI 1.02 to 2.60) for presence of depressive symptoms and 1.50 (95\% CI 0.83 to 2.72 ) for major depression at 12 months. Gender differences were not significant. $12 \% \quad(n=465)$ were unemployed at 6 months. The adjusted OR of being unemployed at 6 months, for participants with major depression at baseline and 6 months was 1.63 (95\% CI 1.01 to 2.64). The OR of unemployment at 24 months associated with major depression at baseline or 6 months in men was 3.52 (95\% CI 0.97 to 12.75 ) adjusted for lifetime depression and was further attenuated on adjustment for prior employment. In women the equivalent OR was 0.98 (95\% CI 0.54 to 1.79 ).

Discussion There is some evidence that both causation and health selection result in raised levels of depressive symptoms in the unemployed. General practitioners should carefully monitor recently unemployed adults for onset of depressive symptoms and possible subsequent major depression. Adults with depression may have raised risks of subsequent unemployment and so should be supported at work.

\section{THE INCIDENCE AND REPETITION OF HOSPITAL-TREATED DELIBERATE SELF-HARM IN IRELAND}

doi:10.1136/jech.2010.120956.25

${ }^{1}$ I J Perry, ${ }^{2} \mathrm{P}$ Corcoran, ${ }^{1} \mathrm{~A}$ P Fitzgerald, ${ }^{3} \mathrm{H}$ S Keeley, ${ }^{2,4} \mathrm{U}$ Reulbach, ${ }^{2} \mathrm{E}$ Arensman. ${ }^{1}$ Department of Epidemiology and Public Health, University College Cork, Republic of Ireland; ${ }^{2}$ National Suicide Research Foundation, Cork, Republic of Ireland; ${ }^{3}$ Child and Adolescent Mental Health Services, Mallow, County Cork, Republic of Ireland; ${ }^{4}$ Department of Public Health and Primary Care, Trinity College Centre for Health Sciences, Adelaide and Meath Hospital incorporating the National Children's Hospital, Tallaght, Dublin, Republic of Ireland

Background Deliberate self-harm (DSH) is one of the strongest risk factors for suicide and a significant public health problem in its own right. Nationally representative data on the incidence, repetition and determinants of DSH are sparse.

Objective To establish the incidence and risk of repetition of hospital-treated DSH in Ireland.

Setting The Republic of Ireland.

Design Surveillance of presentations to hospital emergency departments as part of the Irish National Registry of Deliberate Self Harm.

Participants Individuals who presented with DSH to one of the forty hospital emergency departments that operated in Ireland in 2003-2008. Case ascertainment and data collection were performed by trained data registration officers working independently of the hospitals and following standard operating procedures.

Main outcome measures Annual age-specific and age-adjusted rates of persons presenting to hospital per 100000 population. KaplanMeier cumulative incidence curves and corresponding life-tables showing the risk of repeat DSH presentation at various time intervals and hazard ratios arising from multivariate Cox regression.

Results For 2003-2008, 63154 DSH presentations were recorded involving 41205 individuals. The average annual total, male and female rate of persons presenting with DSH were 202, 172 and 234 per 100000 , respectively. Overall, the female rate was 36\% higher than the male rate. There was a clear peak in the female rate in 15-19 year-olds (651/100000), twice the equivalent rate in men $(323 / 100000)$. In men, the highest rate was in the $20-24$ year age group (426/100 000). For both genders, rates fell with increasing age. Of the 41205 DSH patients, 8755 (21.2\%) presented on at least two occasions. The risk of repetition was highest soon after a DSH presentation. Half $(50 \%)$ of all repeat events occurred within three months. Risk of repetition within 12 months was almost $40 \%$ in subjects who self-cut compared to approximately $25 \%$ for those using other methods of DSH. Repetition rates were similar in men and women. Repetition rates rose rapidly with the number of previous DSH presentations. Respectively, twelve-month repetition rates of $13.7 \%, 36.1 \%, 47.7 \%, 59.7 \%$, and $70.9 \%$ were observed for those with one, two, three, four and five previous presentations.

Conclusion Population-based data on the incidence and repetition of hospital-treated DSH represent an important indicator of the burden of mental illness and the pattern and distribution of suicide risk in the community. The development and implementation of effective methods to reduce repetition rates is an important challenge for health systems.

\section{Births cohorts
026 THE ALL IRELAND TRAVELLER BIRTH COHORT STUDY: OVERCOMING RECRUITMENT CHALLENGES TO ESTIMATE BIRTH PARAMETERS

doi:10.1136/jech.2010.120956.26

N A Hamid, S Abdalla, J Turner, B Quirke, R Niccharthaigh, C Kelleher, P Fitzpatrick, for the All Ireland Traveller Health Study Group. School of Public Health, Physiotherapy and Population Science, University College Dublin, Republic of Ireland

Objective Irish Travellers are a significant minority group characterised by a nomadic lifestyle, specific culture, and significant socioeconomic and health disadvantage. When last documented in 1986, their fertility rate was one of the highest in Europe and infant mortality was almost 3 times the Irish average. The purpose of this study was to investigate prospectively the health status, health utilisation and health care needs of Traveller infants from birth through first year of life.

Setting The Traveller Birth Cohort Study is a linkage sub-study of the All Ireland Traveller Health Study (AITHS) on Island of Ireland (IOI): Republic of Ireland (ROI) and Northern Ireland (NI).

Participants All proband babies born to self-identifying Traveller mothers over a one year period $\left(13^{\text {th }}\right.$ October 2008 to $14^{\text {th }}$ October 2009) on IOI

Methodology A two-stage recruitment process was undertaken through families themselves during the main census using an innovative audio-visual computer-based survey and subsequently consents for the study were obtained by the public health nurses (PHN) or the main study coordinator. In ROI, identification of participants was aided by birth notification forms, the national metabolic screening registers, PHNs' local knowledge and Traveller health projects, and in NI, by Health Visitor notifications and informal local Traveller networks. The participating mothers carry a specifically designed Parent-held Child Record. Fertility rates calculation used data from between the cohort and the AITHS census. Results 980 eligible mothers were identified; to date 468 (51.2\%) mothers consented in ROI and $34(50.7 \%)$ in NI (in progress). Median maternal age was 25cyears old (mean 25.8, SD 5.6) compared to the 2007 national average of 31.1 ; with $75 \%$ of the cohort group under 30 years old. Peak age-group for Travellers was 20-24 years old, this was 30-34 years old for general population. 980 babies were identified, 14 were twins and $51.5 \%$ were male. Since 1986, the Travellers (ROI) crude birth rate has dropped from 34.9 to 25.1; the general- and total period-fertility rates have also dropped from 164.1 to 97.0 and from 5.3 to 2.9 respectively. Both are still significantly above the $2008 \mathrm{ROI}$ national average of 64.6 and 2.1. 